\title{
Analysis of vibration behavior of damped tool arbor by finite element method
}

\author{
Hideaki ONOZUKA*, Koji UTSUMI*, Ippei KONO*, Tomu KATO**, Hayato TAKAHASHI*** \\ and Toshiyuki OBIKAWA**** \\ *Hitachi, Ltd., Yokohama Research Laboratory \\ 292 Yoshida-cho, Totsuka-ku, Yokohama-shi, Kanagawa 244-0817, Japan \\ E-mail:hideaki.onozuka.cf@hitachi.com \\ **Hitachi, Ltd., Tsuchiura works \\ 603 Kandatsu-cho, Tsuchiura-shi, Ibaraki 300-0013, Japan \\ ${ }^{* * *}$ Hitachi Tool Engineering, Ltd. \\ 13-2 Shinizumi, Narita-shi, Chiba 286-0825, Japan \\ **** Institute of Industrial Science, The University of Tokyo \\ 4-6-1 Komaba, Meguro-ku, Tokyo 153-8505, Japan
}

Received 11 January 2014

\begin{abstract}
To improve cutting performance and suppress chatter vibration during machining of large mechanical parts with a long slender cutting tool, a design method was investigated for a tuned mass damper imbedded tool arbor. To clarify the effects of the dynamic stiffness and damping ratio of the weight which is supported at both ends inside the hollow space of the arbor on the dynamic compliance at the end of the arbor, finite element analysis was conducted. First, since the weight has twisting vibration in its 2nd order natural mode, it is necessary to consider 2 degrees of freedom of the motion to optimize the damper. Then, the relationship between the motion of the damper and damping performance is clarified in accordance with the change in spring constant of the weight. From the results, an area exists where there is little change in the maximum negative real part of the compliance due to the change in spring constant. Designing the spring constant in this area stabilizes the damping performance. Furthermore, comparison of the results of finite element analysis and the conventional particle model analysis revealed that the results of finite element analysis show a smaller real part of the compliance than those of particle model analysis. This is because the inertial forces of the weight are applied not to the end of the arbor but to the tool holder side of the arbor and the damping effect is smaller than that in the particle model.
\end{abstract}

Key words : Damped arbor, Mass damper, Machining, Chatter, Optimal design, Finite element method

\section{Introduction}

Machining efficiency has been dramatically improved by the recent increase in the feed speed of machine tools and improvement in the cutting performance of tool materials. However, during the machining of large mechanical parts like the cavity shape of dies and molds, the materials need to be machined by using long, slender cutting tools. By using a long cutting tool in the machining process, chatter vibration easily occurs due to the deterioration of the dynamic stiffness of the cutting tool. Chatter vibration deteriorates the roughness of the machined surface and damages the cutting edge of the tool. The chatter vibration is affected by spindle speed, depth of cut, dynamic stiffness of the cutting tool or work material, and material property of the work piece as shown in Fig. 1. To avoid the chatter vibration, it is effective to decrease the cutting conditions such as spindle speed and depth of cut or increase dynamic stiffness of mechanical structure. There are two ways to improve the dynamic stiffness of the mechanical structure such as increasing static stiffness or dynamic stiffness namely damping behavior. If those mechanical structures must be made from steel, increases in static stiffness are limited due to the restriction of design geometry. However, methods are 
available for increasing dynamic stiffness by attaching a mass damper into the boring bar or ram of a machine tool (Seto and Yamanaka, 1980, Seto, 2010, Madoliat, et al., 2011, Rashid and Nicolescu, 2008). Moreover, optimal designing and tuning methods have been reported for the mass damper built in an end mill or boring bar (Sims, 2007, Saffury and Altus, 2009, Rivin and Kang, 1992, Pratt and Nayfeh, 2001, Semercigil and Chen, 2002), which, according to their research results, make it possible to avoid chatter vibration and increase cutting efficiency in the machining of deep hole or cavity shape.

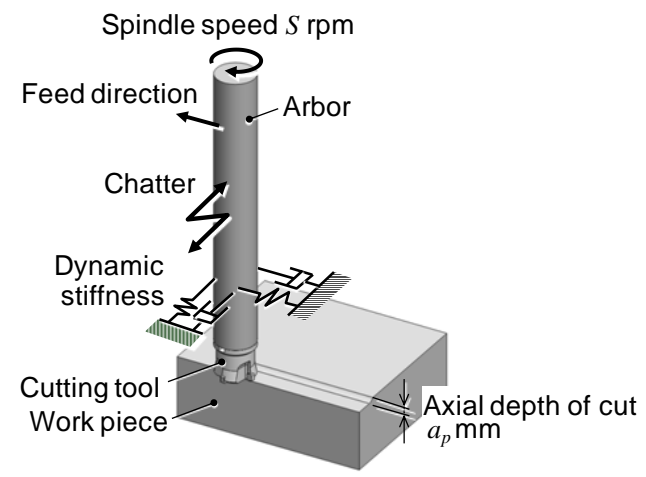

Fig. 1 Chatter vibration in machining process with a long slender tool arbor.

Figure 2 shows a typical structure of a damper which is installed in long cutting tool such as milling tool arbor or boring bar. The weight is supported at the both ends by springs in the hollow space of the arbor. This structure can be modeled as shown in Fig.3. The arbor body and the cutting tool vibrate in radial direction and the weight is connected to the arbor body at the both ends in the hollow space. $m_{\mathrm{d}}, k_{\mathrm{d}}$ and $c_{\mathrm{d}}$ are the modal mass of the weight, the stiffness of the spring, and the damping coefficient of the damper, respectively. The design parameters of the vibration system are optimized by being approximated as a 2 degrees of freedom system of a particle model as shown in Fig.4(Onozuka, et al., 2013). In this model, $m_{1}, k_{1}$, and $c_{1}$ are modal mass, modal stiffness, and modal damping coefficient of the end of cutting tool, respectively. $m_{1}$ and $k_{1}$ of the bending mode of the arbor body vibration can be able to be calculated by using Rayleigh's method. In this figure, spring constant of the damper is $k_{2}=2 k_{\mathrm{d}}$, damping coefficient is $c_{2}=2 c_{\mathrm{d}}$, and mass of the weight is $m_{2}=m_{\mathrm{d}} . x_{1}, x_{2}$ in this figure express the displacement of the arbor body and the weight respectively when the force $f_{1}$ is applied to the end of the arbor body.

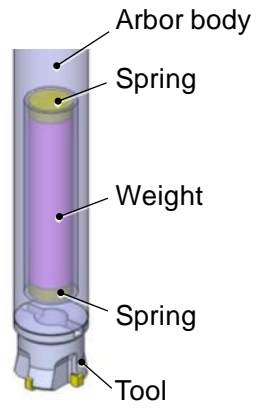

Fig. 2 Mass damper in hollow space of arbor body.

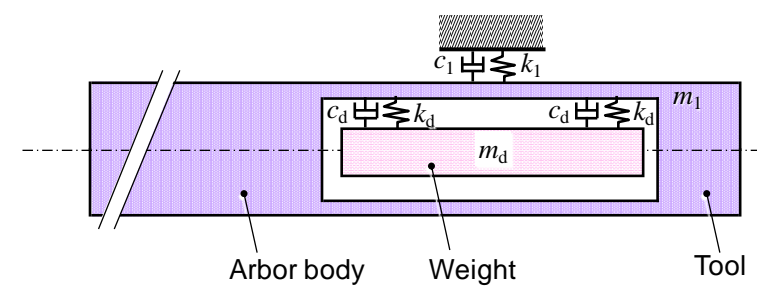

Fig. 3 Vibration model of a damper installed arbor.

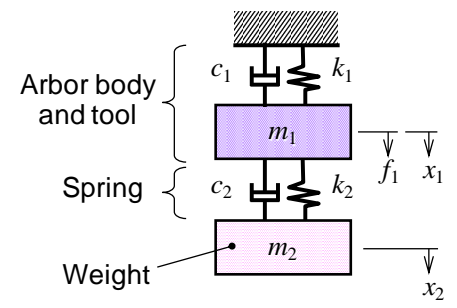

Fig.4 Two degrees of freedom of particle model of the vibration system. 
However, since the actual weight is considered to have a twisting vibration in 2 nd order mode, it must affect the damping performance of the arbor. Additionally, since the weight is connected to the inside of the hollow space of the arbor body, it does not apply inertial force on the end of cutting tool as the particle model shown in Fig.4. In this research, the effects of the vibration behavior of the mass damper on the dynamic stiffness of the cutting tool at its end are investigated in order to optimize the damper. For this objective, first, relationship between the natural vibration mode of the weight and the compliance of the damped arbor was clarified considering multi order mode vibration of the weight and arbor body by using a finite element method. Then optimal conditions of a damper were studied, such as supporting stiffness and damping ratio for maximizing the maximum negative real part of the dynamic compliance of the arbor. Especially, the range of the stiffness of supporting spring of the weight was clarified. Furthermore, analytical results of dynamic stiffness were discussed by comparing them with the results of the particle model.

\section{Modeling and analysis method}

In this research, dynamic stiffness of a tool arbor is analyzed by using finite element analysis software FEX ${ }^{\circledR}$. As shown in Fig.5, the length of the arbor body and cutting tool is $400 \mathrm{~mm}$ and the diameter is $47 \mathrm{~mm}$. The left end of the arbor is BT holder and it is connected to a spindle of a machine tool. The dynamic stiffness of tool side end is affected by not only the flexibility of the long tool arbor, but also the flexibility of the BT holder and the mechanical structure of the machine tool. Fig.6 shows the analytical model. In order to consider the influence of flexibility of the holder and machine tool, material properties of the left end of the model with the length of $50 \mathrm{~mm}$ were given artificially. This part represents the contribution of the holder and machine tool to the dynamic stiffness, and the material properties as Young's modulus and density are defined in accordance with the measured dynamic stiffness at the right end of the arbor. The area of $350 \mathrm{~mm}$ length of the arbor body and tool which is made of chromium molybdenum steel (JIS-SCM435) has a $47 \mathrm{~mm}$ diameter. Density of the arbor body and tool is $7.8 \times 10^{3} \mathrm{~kg} / \mathrm{m}^{3}$, Young's modulus is $2.1 \times 10^{2}$ $\mathrm{GPa}$ respectively. Hollow space is designed with a diameter of $36 \mathrm{~mm}$ and length of $150 \mathrm{~mm}$ in the arbor body.

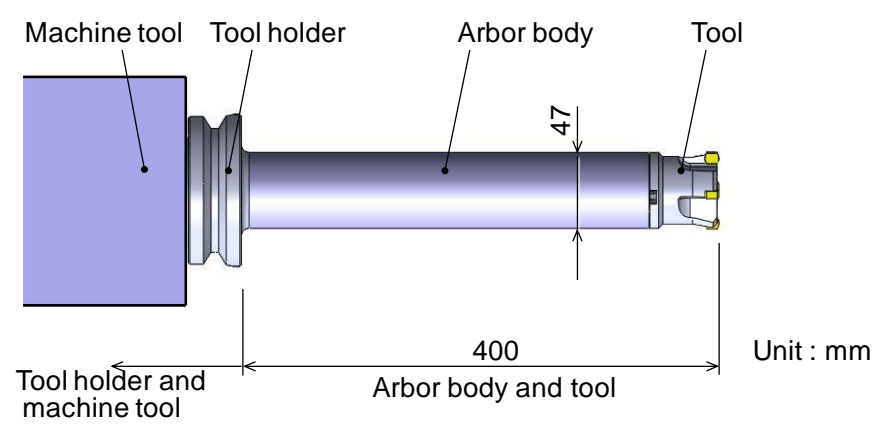

Fig.5 Geometry of objective tool arbor.

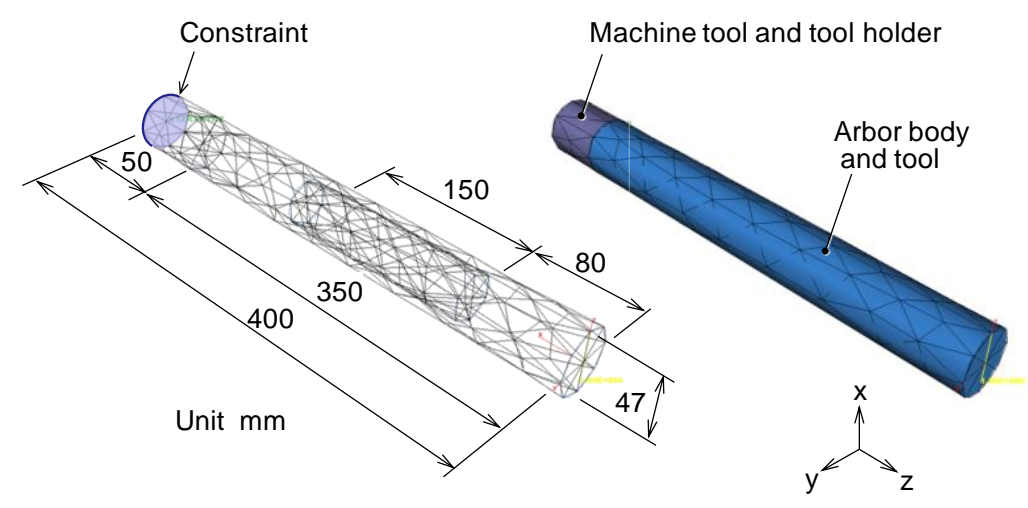

Fig.6 Finite element model of body of tool arbor and tool holder. 
Figure 7 shows the model of the damper which consists of a weight and springs. The weight is supported by springs at both ends. The damper is $34 \mathrm{~mm}$ in diameter, and $130 \mathrm{~mm}$ long. The weight is assumed to be made from high density material such as sintered tungsten, and the spring is made from elastic material such as plastics. To investigate the dynamic behavior of the damper, displacements at both ends of the weight (A and C) and the center (B) were evaluated.

First, so that the maximum amplitude and natural frequency agree with the measured frequency response at the end of the arbor, the material properties of the $50 \mathrm{~mm}$ area at the left end are given artificially. Fig. 8 shows an example of the vibration mode at natural frequency. Fig. 9 shows the measured frequency response of the arbor that is installed in the machine tool via BT50 tool holder. The frequency response calculated by Fourier transform of the force of the impact hammer and the displacement of the accelerometer. Natural frequency of the arbor $f_{\text {measure_l }}$ is $183 \mathrm{~Hz}$ and the damping ratio $\zeta_{\text {measure_1 }}$ is 0.04 on the machine tool. Modal stiffness of the arbor in the natural mode $k_{\text {measure_1 }}$ is $2.08 \times 10^{6} \mathrm{~N} / \mathrm{m}$, mass $m_{\text {measure } 11}$ is $0.678 \mathrm{~kg}$ respectively. This figure presents calculated analytical results as well. From these results, so that the analyzed result agrees with the measured frequency response within 5\% error, it is assumed that the $50 \mathrm{~mm}$ long element in the area at the end of the tool holder side has Young's modulus $1.5 \times 10^{2} \mathrm{GPa}$, the damping ratio $\zeta_{\mathrm{b} 1}=0.05$, and $f_{\mathrm{b} 1}=188 \mathrm{~Hz}$.

In the design of the actual damped tool arbor, the supporting stiffness of the weight, namely spring constant, is regulated by the geometrical shape or material property of the spring. In this research, in order to clarify the effects of the supporting stiffness of the damper on the dynamic stiffness of the arbor, Young's modulus of the spring material was changed. Relationship between the Young's modulus of the spring material and the stiffness of the damper was identified by the finite element analysis. Fig. 10 shows the calculated results of the static stiffness at position B of the damper when Young's modulus of the spring is varied. Static stiffness of the damper is proportional to Young's modulus of the spring. According to this result, the spring constant of the damper $k_{2}$ is expressed as the following equation by using Young's modulus of the spring $E_{\mathrm{s}}$.

$$
k_{2}=60.2 \times 10^{4} \cdot E_{\mathrm{s}}+1.97 \times 10^{4}
$$

Analytical conditions are summarized in Table.1.

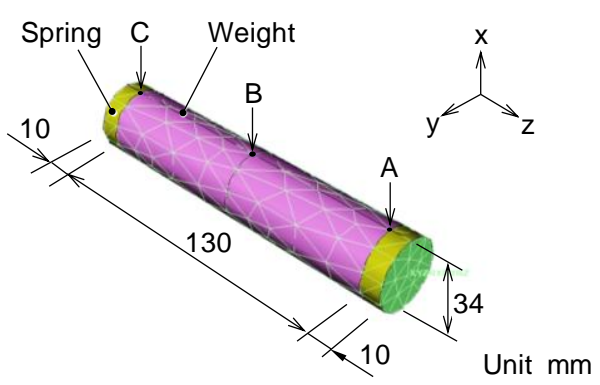

Fig.7 Finite element model of the damper.

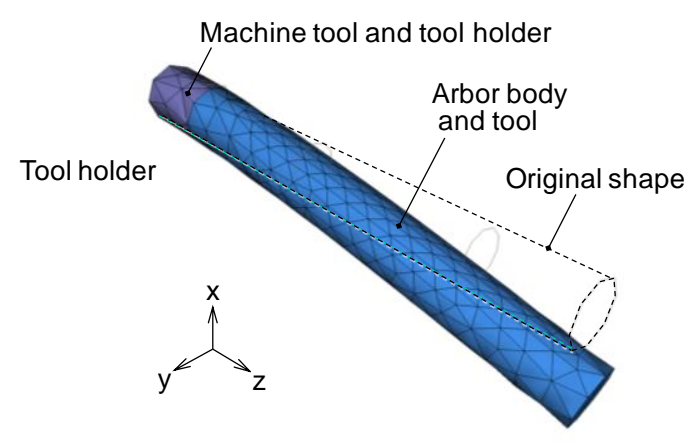

Fig.8 Mode shape of vibration of the body. 


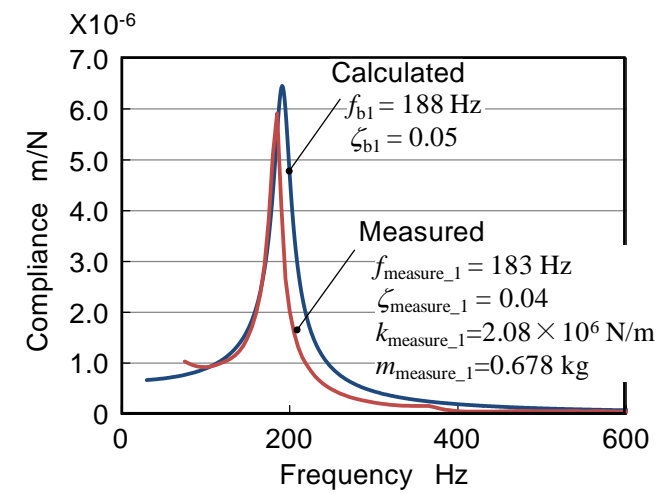

Fig. 9 Approximation of the supporting stiffness of the tool holder.

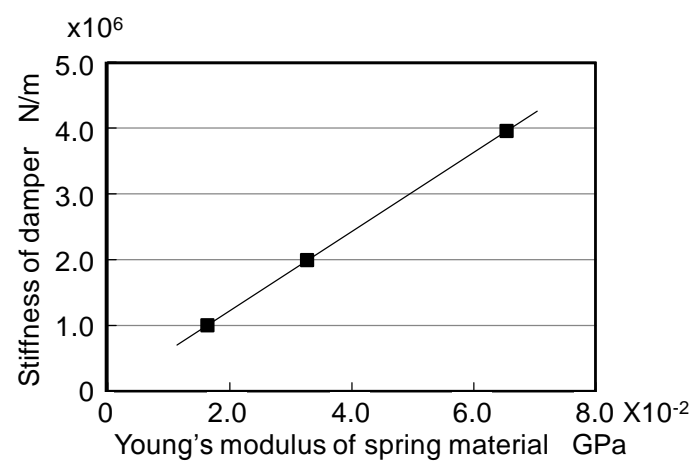

Fig. 10 Relationship between Young's modulus of spring material and static stiffness of damper.

Table.1 Simulation Conditions

\begin{tabular}{l|lll}
\hline & $\begin{array}{l}\text { Density } \\
\mathrm{kg} / \mathrm{m}^{3}\end{array}$ & $\begin{array}{c}\text { Young's modulus } \\
\mathrm{GPa}\end{array}$ & Damping ratio \\
\hline Body & $7.8 \times 10^{3}$ & $2.1 \times 10^{2}$ & 0.05 \\
\hline Tool holder & $7.8 \times 10^{3}$ & $1.5 \times 10^{2}$ & 0.05 \\
\hline Weight & $1.5 \times 10^{4}$ & $5.0 \times 10^{2}$ & 0.05 \\
\hline Spring & $1.0 \times 10^{3}$ & $\begin{array}{l}0.817 \times 10^{-2} \\
-6.536 \times 10^{-2}\end{array}$ & $\begin{array}{l}0.1 \\
-0.3\end{array}$ \\
\hline
\end{tabular}

\section{Analytical results}

\subsection{Dynamic behavior of damper}

Figure 11 shows the motion of the damper at the natural frequency when spring stiffness is $k_{2}=2.0 \times 10^{6} \mathrm{~N} / \mathrm{m}$ and damping ratio $\zeta_{2}$ is 0.05 . The frequency of the 1 st order mode is $f_{\mathrm{d} 1}=159 \mathrm{~Hz}$. As shown in Fig. 9 (a), weight displaces in the $\mathrm{x}$ direction. The 2 nd vibration mode at $239 \mathrm{~Hz}$ is the twisting motion around y-axis as shown in Fig. 9 (b).

Fig. 12 shows the relationship between the spring constant of the weight supporting spring and natural frequency. The frequencies of the natural vibration for the 1st order mode and 2nd order mode are increased by the increase in the stiffness of damper. If the vibration of the 1st order mode is assumed to be a single degree of freedom system as shown in Fig. 13(a), natural frequency of the 1 st order mode $f_{\mathrm{d} 1}$ is expressed as the following equation.

$$
f_{\mathrm{d} 1}=\frac{1}{2 \pi} \sqrt{\frac{k_{2}}{m_{2}}}
$$

On the other hand, the 2nd order mode of the damper is assumed to be twisting vibration as shown in Fig. 13(b). The weight is supported at both ends by springs that have a spring constant of $k_{2} / 2$. Twisting stiffness $K_{2}$, the moment of inertia of the cylindrical weight around y-axis $J_{2}$, is expressed as follows;

$$
K_{2}=2 \cdot \frac{k_{2}}{2} \cdot\left(\frac{l_{2}}{2}\right)^{2}
$$




$$
J_{2}=\left(\frac{1}{4} \cdot\left(\frac{d_{2}}{2}\right)^{2}+\frac{1}{12} \cdot l_{2}^{2}\right) \cdot m_{2}
$$

where $d_{2}$ is the diameter and $l_{2}$ is the length of the weight.

Frequency of natural vibration of twisting mode $f_{\mathrm{d} 2}$ is expressed as follows;

$$
f_{\mathrm{d} 2}=\frac{1}{2 \pi} \sqrt{\frac{K_{2}}{J_{2}}}
$$

By using these equations, natural frequencies $f_{\mathrm{d} 1}$ and $f_{\mathrm{d} 2}$ are calculated where the mass of the weight $m_{2}=1.77 \mathrm{~kg}$ as indicated in Fig.12. As shown in this figure, frequencies of the 1st and 2nd order modes of natural vibration agree well to the calculated results.

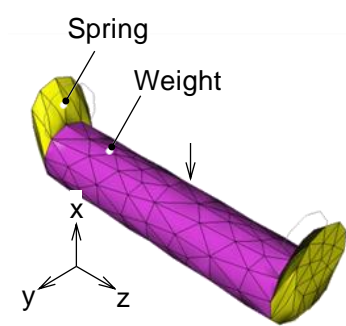

$159 \mathrm{~Hz}$

(a) 1st order mode (Parallel mode)

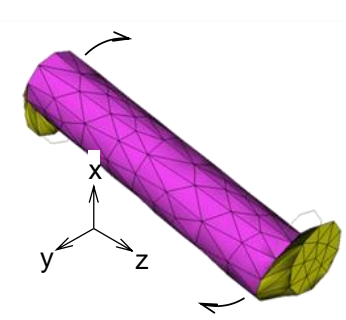

$293 \mathrm{~Hz}$

(b) 2nd order mode (Twist mode)

Fig. 11 Mode shapes of the damper vibration.

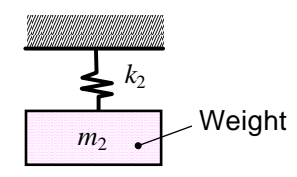

(a) 1st order mode

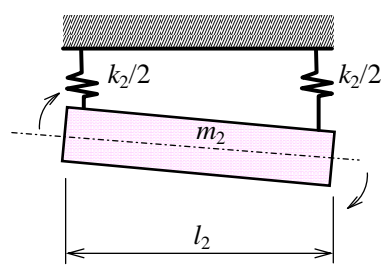

(b) 2nd order mode

Fig. 13 Vibration mode of the weight.

Next, dynamic stiffness of the damper is evaluated by finite element analysis. Regenerative chatter (Tlusty and Polacek, 1963, Tobias, 1965) and mode coupling (Ota, et al., 2007, Kondo, et al., 1998, Zhang, et al., 2012, Suzuki, et al., 2009) are known as factors of self excited chatter vibration. In this research, chatter suppression performance is assumed to be evaluated by the maximum negative real part value of the compliance by average tooth angle approach (Tlusty, 1985) in order to establish the optimal design method for a damped tool arbor. Fig. 14 shows the real part of the compliance at position $\mathrm{B}$, which is the center of the weight, when impact force is applied at position $\mathrm{A}$ in the $\mathrm{X}$ direction. Although vibration of the weight has two modes in $\mathrm{x}-\mathrm{Z}$ plane as shown in Fig.11, displacement at position $\mathrm{B}$ is very small at $293 \mathrm{~Hz}$ since the weight rotates around the center of the weight at the frequency of the 2 nd mode.

Figure 15(a) presents the real part of the dynamic compliance when impact force is applied to position $\mathrm{A}$ in the $\mathrm{x}$ direction. At position A, frequency responses at both $159 \mathrm{~Hz}$ and $293 \mathrm{~Hz}$ are in the same phase since the displacement is in the same direction. On the other hand, the real part of the compliance is negative at $f_{\mathrm{d} 2}=293 \mathrm{~Hz}$, which is the natural frequency of the 2nd mode as shown in Fig.15 (b) when the impact force is applied to position A. Position C displaces in the opposite direction because the weight rotates around its center in the 2nd mode.

As described above, since the damper that has weight supported at both ends by springs applies different force to the arbor 
body in the 2nd natural frequency mode, 2 degrees of freedom of the vibration of the damper must be considered in the analysis.

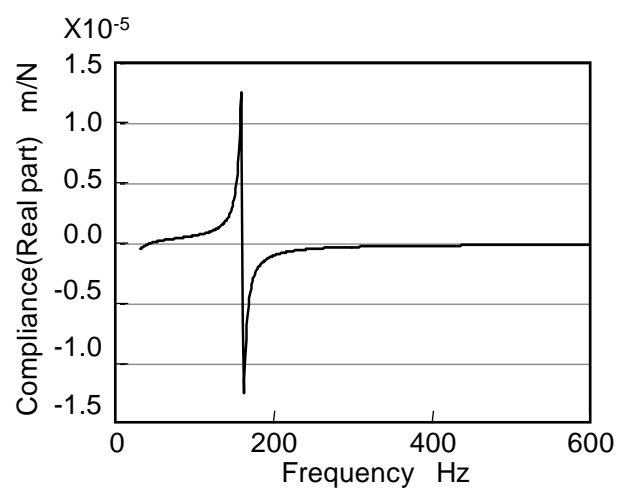

Fig. 14 Compliance of the damper vibration at position B when impact force is applied at position A.

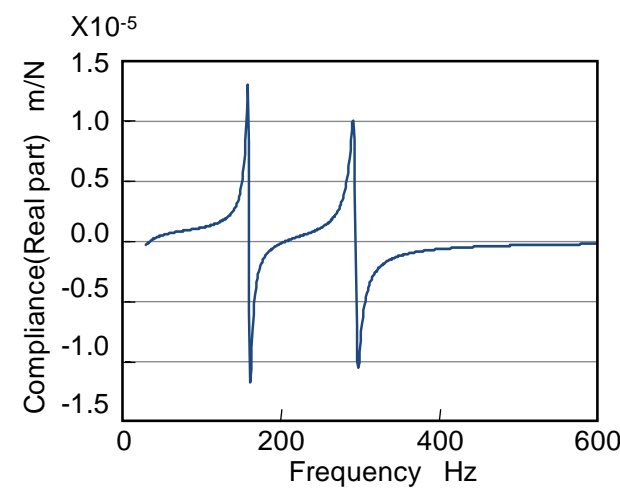

(a)Position A

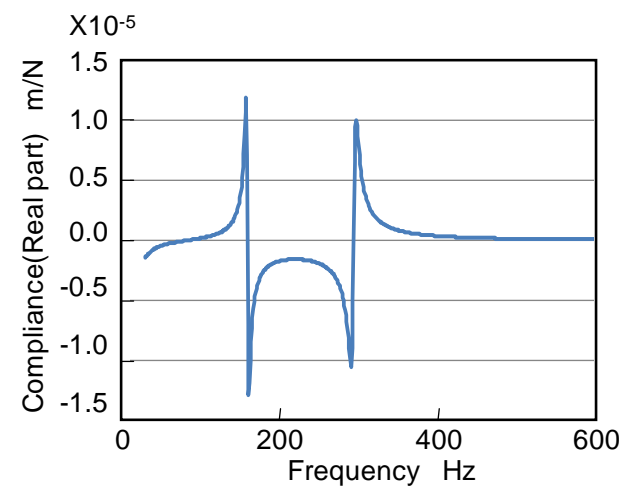

(b)Position C

Fig.15 Compliance of damper vibration at position A and $\mathrm{C}$ when impact force is applied at position A.

\subsection{Relationship between the vibration behavior of the damper and the dynamic stiffness of the damped arbor}

Dynamic stiffness of the damped arbor in which damper as shown in Fig.5 is installed in its hollow space is analyzed. Fig. 16 shows the real part of dynamic stiffness under conditions in which the spring constant of the damper is $k_{2}=0.5$ $\times 10^{6} \mathrm{~N} / \mathrm{m}$ and the damping ratio is $\zeta_{2}=0.3$. The figure of the vibration mode shows the displacement and deformation of the arbor body, spring, and weight when the displacement of the arbor body is maximized in the $\mathrm{x}$ direction. In this figure, relative displacement of the nodal points of the analytical model is drawn by being expanded, and this figure exaggerates the relative displacement and deformation of each part of the damped arbor. The frequencies of three natural vibration modes are also indicated. These modes are different from the natural modes of the arbor body or damper. This figure shows that the frequency of the 1st order natural vibration mode of the system is $82 \mathrm{~Hz}$. In this vibration mode, weight displaces in the negative $\mathrm{x}$ direction, which is the same phase of the arbor body. At $157 \mathrm{~Hz}$ and $198 \mathrm{~Hz}$, which is close to 2nd order natural frequency of the damper $f_{\mathrm{d} 2}$, motion of the weight is rotating around y-axis. The end of the weight displaces in the same direction as the arbor body at $157 \mathrm{~Hz}$ and in the opposite direction at 198 Hz.

Figure 17 shows the analytical results where the spring constant of the damper $k_{2}$ is $1.5 \times 10^{6} \mathrm{~N} / \mathrm{m}$ and damping ratio $\zeta_{2}$ is 0.3 . In this case, natural frequency of the damper is $f_{\mathrm{d} 1}=146 \mathrm{~Hz}$. In this 1 st order mode of the damped arbor at the frequency of $122 \mathrm{~Hz}$, weight displaces in the same direction. At the 2nd order mode of the damped arbor at the frequency of $217 \mathrm{~Hz}$, which is close to the natural frequency of the arbor body, weight displaces in the opposite phase to the arbor body. At $282 \mathrm{~Hz}$, which is the 3rd order mode of the damped arbor, weight rotates around y-axis, and its tool side end displaces in the opposite direction to the tool. 
Dynamic stiffness of the arbor is analyzed for larger spring constant $k_{2}$ of the damper. Fig. 18 shows the results for spring constant $k_{2}=3.0 \times 10^{6} \mathrm{~N} / \mathrm{m}$ and damping ratio $\zeta_{2}=0.3$. In this case, natural frequency of the damper is $207 \mathrm{~Hz}$. Although the natural frequency of the damped tool is different from that in Fig.16, the vibration mode is similar.

As expressed above, the negative real part of the dynamic stiffness maximizes when the spring constant of the damper $k_{2}$ is $1.5 \times 10^{6} \mathrm{~N} / \mathrm{m}$ as shown in Fig. 17 .

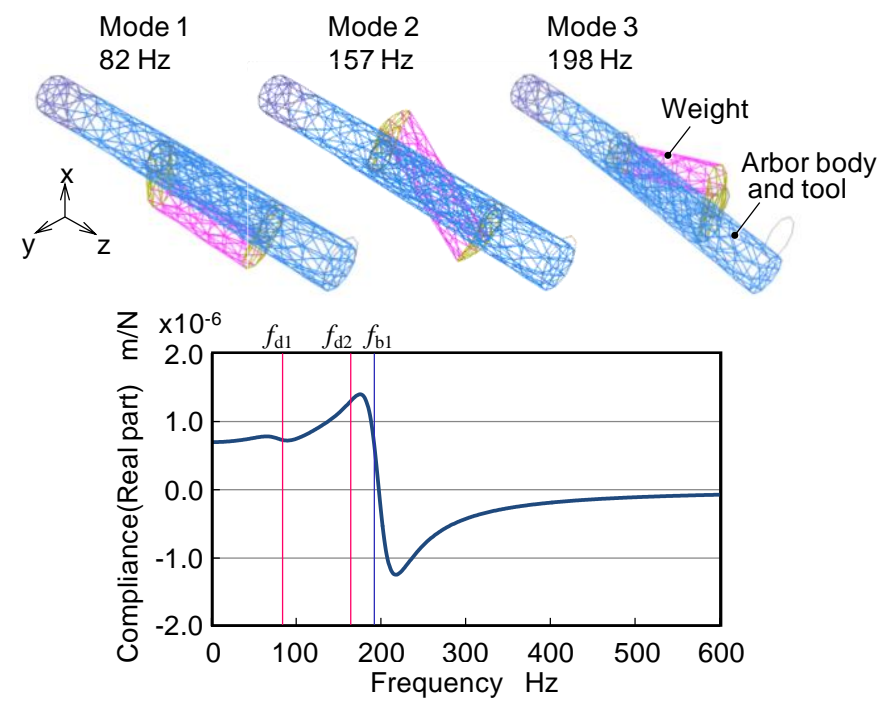

Fig.16 Mode shape and dynamic stiffness of arbor when stiffness of damper is $k_{2}=0.5 \times 10^{6} \mathrm{~N} / \mathrm{m}$ and $\zeta_{2}=0.3$.

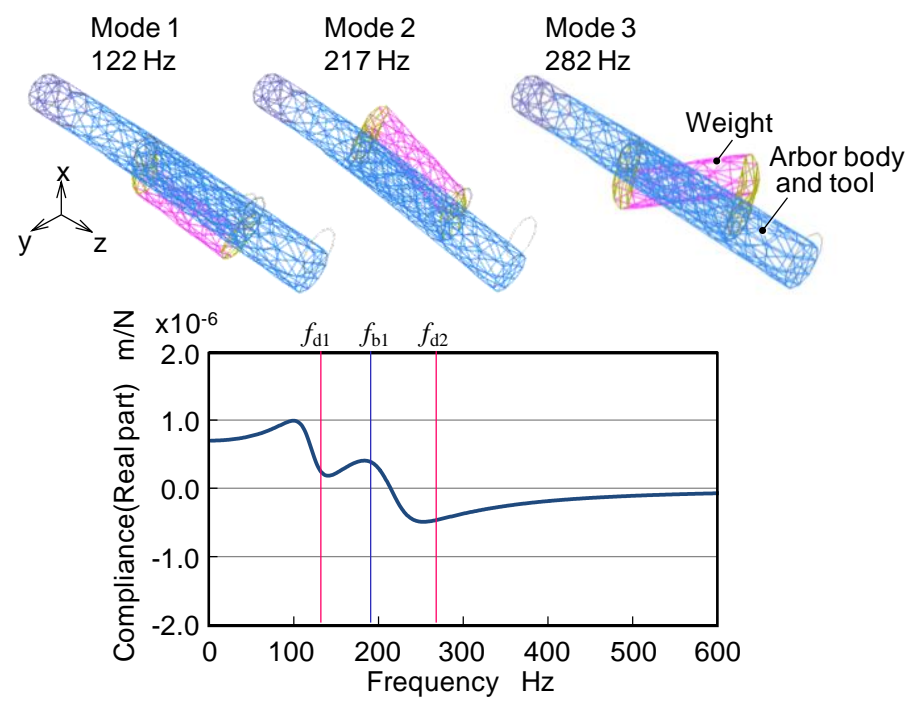

Fig.17 Mode shape and dynamic stiffness of arbor when stiffness of damper is $k_{2}=1.5 \times 10^{6} \mathrm{~N} / \mathrm{m}$ and $\zeta_{2}=0.3$. 


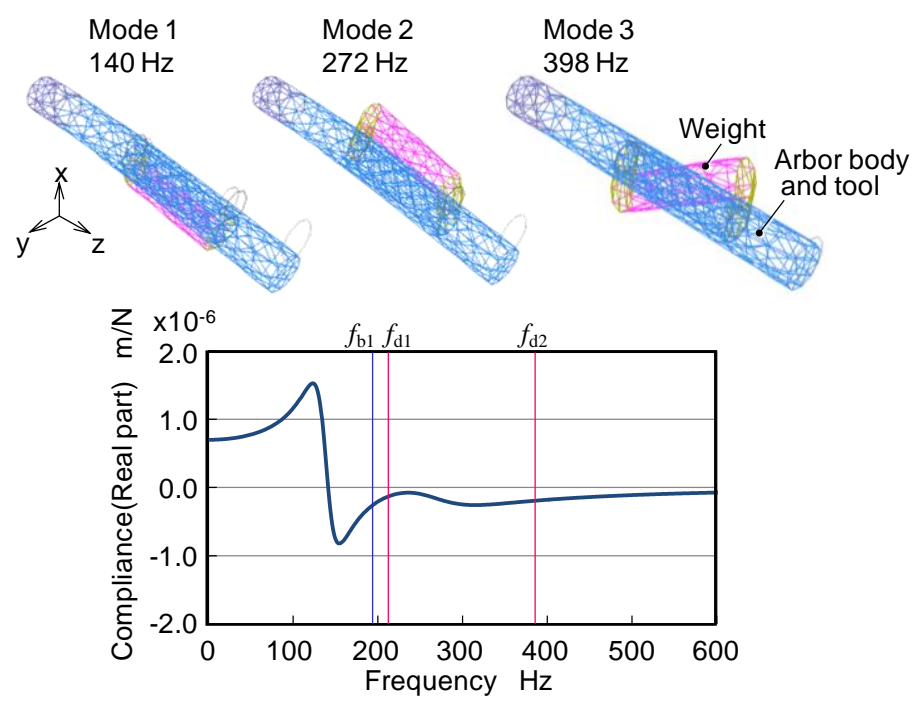

Fig.18 Mode shape and dynamic stiffness of arbor when stiffness of damper is $k_{2}=3.0 \times 10^{6} \mathrm{~N} / \mathrm{m}$ and $\zeta_{2}=0.3$.

\subsection{Optimization of the damper conditions}

Dynamic stiffness is analyzed by changing the spring constant $k_{2}$ from $0.5 \times 10^{6} \mathrm{~N} / \mathrm{m}$ to $4.0 \times 10^{6} \mathrm{~N} / \mathrm{m}$ and the damping ratio of damper $\zeta_{2}$ from 0.2 to 0.4 . Fig. 19 shows the maximum negative real part of the analyzed damped tool which has $47 \mathrm{~mm}$ diameter and 400mm length.

This figure shows that when the spring constant $k_{2}$ is $2.0 \times 10^{6} \mathrm{~N} / \mathrm{m}$, compliance is maximum. The maximum negative real part of the compliance also increases due to the increase in the damping ratio, but the optimal spring constant does not show much change. Moreover, although the maximum negative real part changes little in the area of $k_{2}$ from $1.0 \times 10^{6} \mathrm{~N} / \mathrm{m}$ to $2.0 \times 10^{6}$ $\mathrm{N} / \mathrm{m}$, it deteriorates steeply in other areas.

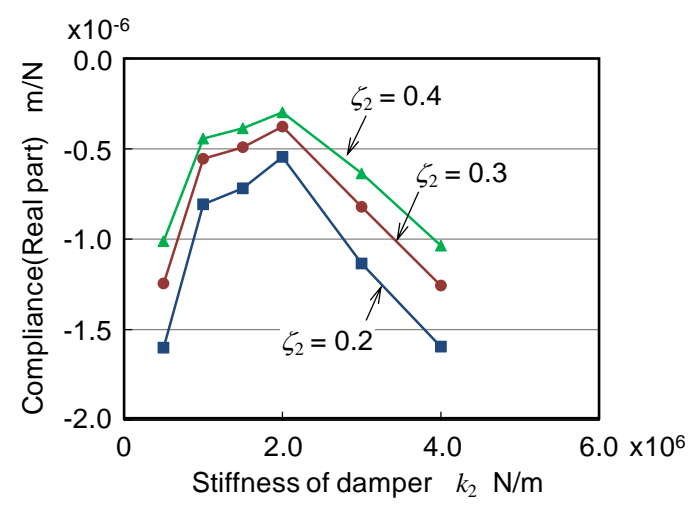

Fig. 19 Relationship between stiffness of damper and dynamic stiffness.

\section{Discussion}

\subsection{Relationship between the vibration mode of the damper and the compliance of the arbor}

As shown in Fig. 16, if $f_{\mathrm{d} 1}<f_{\mathrm{d} 2}<f_{\mathrm{b} 1}$, the vibration mode of the damper is twisting around $198 \mathrm{~Hz}$, which is close to the natural frequency of the arbor body. The tool side end of the weight applies inertial force to the arbor body by the twisting motion. When $f_{\mathrm{d} 1}<f_{\mathrm{b} 1}<f_{\mathrm{d} 2}$ as shown in Fig. 17, the 1 st order mode of the natural vibration of the damper that is parallel motion of the weights attenuates the vibration of arbor effectively. Although the 2nd order mode of the damper may also affect the damping performance, since the weight rotates around its center, damping performance is considered to be worse than in the case in Fig. 
16. As shown in Fig.18, when $f_{\mathrm{b} 1}<f_{\mathrm{d} 1}<f_{\mathrm{d} 2}$, the 1 st order mode of the damper which is parallel motion of the weight is not able to apply enough inertial force to the arbor body and damping performance is poor. From these results, It is found that natural frequency of damper must satisfy $f_{\mathrm{d} 1}<f_{\mathrm{b} 1}<f_{\mathrm{d} 2}$ in order to maximize the performance of the damper.

\subsection{Optimal condition of the damper}

Figure 20 shows the relationship between the ratio of natural frequency of the arbor body and damper $f_{\mathrm{d} 1} / f_{\mathrm{b} 1}$ and maximum negative real part of the compliance. Damping performance is maximized by assuming the natural frequency ratio $f_{\mathrm{d} 1} / f_{\mathrm{b} 1} 0.83$. An area was found where the change in compliance was small. If the frequency ratio is $0.59<f_{\mathrm{d} 1} / f_{\mathrm{b} 1}<0.83$ as shown in this figure, stable damping performance is obtained.

Figure 21 shows the relationship between the frequency ratio and calculated magnitude of compliance. In these results, optimal frequency ratio $f_{\mathrm{d} 1} / f_{\mathrm{b} 1}$ is 0.72 , although it is 0.83 in the results of the maximum negative real part of the compliance. From the results in Fig.18 and Fig.19, optimal frequency ratio differs almost 15\% by changing the evaluation method for dynamic stiffness.

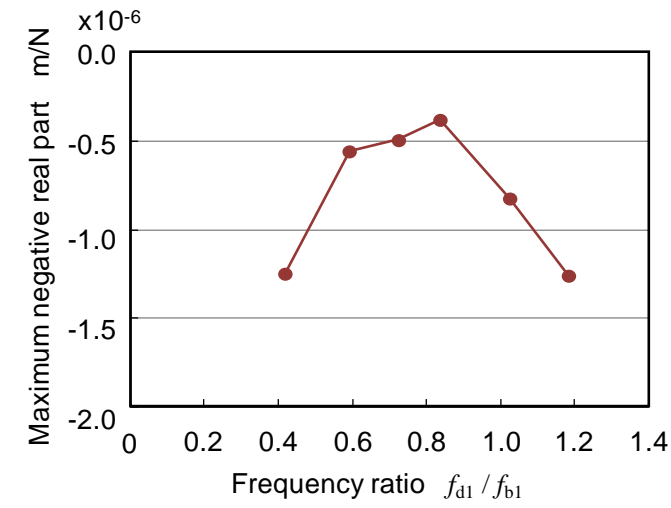

Fig.20 Relationship between frequency ratio and maximum negative real part of compliance.

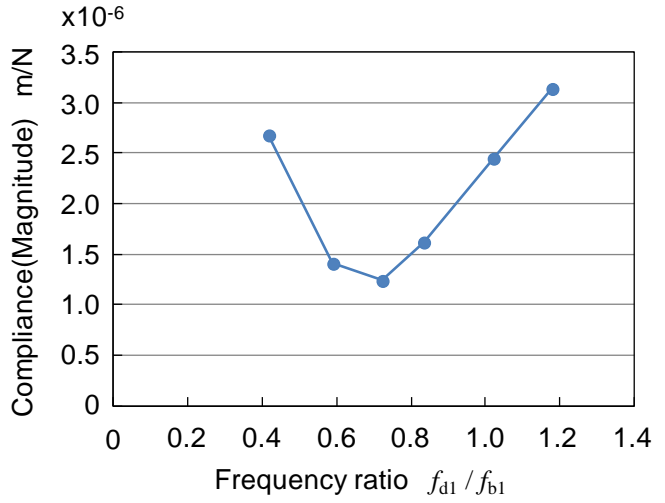

Fig. 21 Relationship between frequency ratio and maximum negative real part of compliance

\subsection{Comparison of the results of finite element method and particle model}

To validate the effects of finite element analysis that considers multi degrees of freedom of the vibration behavior of the damper, its results are compared with those calculated by using the particle system model. As shown in Fig.4, both the arbor body and damper are assumed to be particles. The equation of the motion is expressed as follows for modal parameters of the arbor body $m_{1}, k_{1}, c_{1}$, and the damper $m_{2}, k_{2}, c_{2}$.

$$
\begin{aligned}
& k_{1} x_{1}+c_{1} \dot{x}_{1}+m_{1} \ddot{x}_{1}-k_{2}\left(x_{2}-x_{1}\right)-c_{2}\left(\dot{x}_{2}-\dot{x}_{1}\right)=f_{1} \\
& k_{2}\left(x_{2}-x_{1}\right)+c_{2}\left(\dot{x}_{2}-\dot{x}_{1}\right)+m_{2} \ddot{x}_{2}=0
\end{aligned}
$$

where $x_{1}$ and $x_{2}$ are the displacements of the arbor body and the damper, respectively. Specifically,

$$
\left[\begin{array}{cc}
k_{1}+k_{2} & -k_{2} \\
-k_{2} & k_{2}
\end{array}\right]\left\{\begin{array}{l}
x_{1} \\
x_{2}
\end{array}\right\}+\left[\begin{array}{cc}
c_{1}+c_{2} & -c_{2} \\
-c_{2} & c_{2}
\end{array}\right]\left\{\begin{array}{l}
\dot{x}_{1} \\
\dot{x}_{2}
\end{array}\right\}+\left[\begin{array}{cc}
m_{1} & 0 \\
0 & m_{2}
\end{array}\right]\left\{\begin{array}{c}
\ddot{x}_{1} \\
\ddot{x}_{2}
\end{array}\right\}=\left\{\begin{array}{c}
f_{1} \\
0
\end{array}\right\}
$$

Equation (8) is expressed as,

$$
[K]\{X\}+[C]\{\dot{X}\}+[M]\{\ddot{X}\}=\{F\}
$$

In Laplace domain, 
$\left([K]+j \omega[C]-\omega^{2}[M]\right)\{X\}=\{F\}$

Then,

$$
\{X\}=\left([K]+j \omega[C]-\omega^{2}[M]\right)^{-1}\{F\}
$$

Therefore the compliance of the vibration system is given by;

$$
[\phi]=\left([K]+j \omega[C]-\omega^{2}[M]\right)^{-1}
$$

By equation (12), dynamic stiffness of the arbor, dynamic compliance of the arbor is calculated under conditions in which the damping ratio of the damper is $\zeta_{2}=0.3$, modal mass of the arbor is $m_{1}=0.678 \mathrm{~kg}$, modal stiffness is $k_{1}=2.08 \times$ $10^{6} \mathrm{~N} / \mathrm{m}$, and modal damping ratio is $\zeta_{1}=0.04$ from measurement results shown in Fig.9.

Additionally, damping constant $c_{1}, c_{2}$ are;

$$
c_{1}=2 \sqrt{m_{1} k_{1}} \zeta_{1}, c_{2}=2 \sqrt{m_{2} k_{2}} \zeta_{2}
$$

Figure 22 shows the maximum negative real part of the compliance for various spring constants $k_{2}$ by using this equation. This figure also shows results obtained by the finite element method. The maximum negative real part of the compliance for the change in spring constant of the damper shows a similar tendency, but the optimal spring constant is different. Compliance of the finite element analysis maximizes when the spring constant $k_{2}$ is $k_{2}=2.0 \times 10^{6} \mathrm{~N} / \mathrm{m}$ whereas $k_{2}=1.5 \times 10^{6} \mathrm{~N} / \mathrm{m}$ is optimal for the particle model. The maximum negative real part of the compliance for optimal spring constant of the particle model is larger than that of the finite element method.

In either case, change in the compliance is small in the area close to the optimal spring constant. Therefore, designing the spring constant of the damper in this area achieves stable damping performance.

In the particle model, inertial force of the weight is assumed to be applied to the end of the arbor. However, in the finite element model, the damper is connected at the positions $80 \mathrm{~mm}$ and $150 \mathrm{~mm}$ from the end of the arbor. Therefore, the damping performance of the damper becomes poor, and the maximum negative real part of the compliance is smaller as shown in Fig.22.

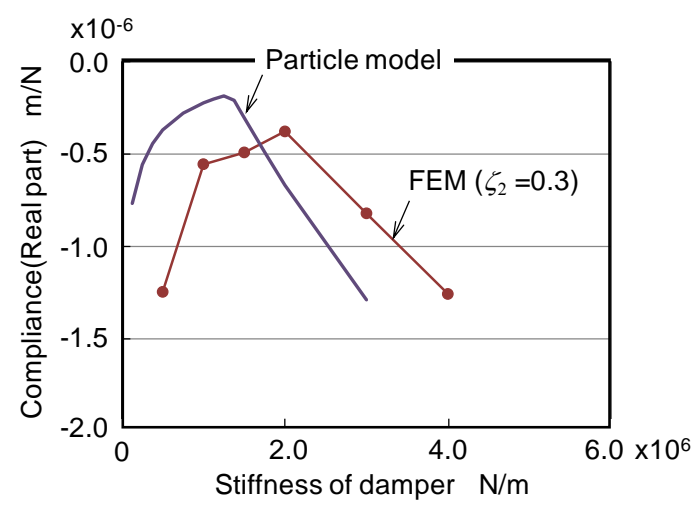

Fig. 22 Comparison of dynamic stiffness calculated by FEM and particle model.

\section{Conclusions}

To improve cutting performance and suppress chatter vibration during machining of large mechanical parts, a design method was investigated for a tuned mass damper imbedded tool arbor. To clarify the effects of vibration behavior of the damper on the compliance at the tool end and obtain the optimal design method, finite element analysis was conducted and revealed the following key results.

(1)Since the weight supported at both ends and it has the 2nd order natural vibration mode, which is a twisting motion, 
2 degrees of freedom of the weight must be considered to optimize the damper. From the finite element analysis, natural frequency of the arbor body $f_{\mathrm{b} 1}$, natural frequency of the damper $f_{\mathrm{d} 1}$ and $f_{\mathrm{d} 2}$ which are 1 st and 2 nd order mode respectively must satisfy $f_{\mathrm{d} 1}<f_{\mathrm{b} 1}<f_{\mathrm{d} 2}$ in order to optimize the performance of the damper.

(2)An area of the spring constant of the damper exists where there is little change in the maximum negative real part of the compliance due to the change in spring constant of the damper. Designing the spring constant of the damper in this area achieves stable damping performance. In the case of the arbor with the length of $400 \mathrm{~mm}$, frequency ratio of $0.59<f_{\mathrm{d} 1} / f_{\mathrm{b} 1}<0.83$ gives stable damping performance due to the change of spring constant of the damper.

(3)Results of finite element analysis show a smaller real part of the compliance than particle model analysis since the inertial forces of the weight are applied not to the end of arbor but to the tool holder side of the arbor and the damping effect is smaller than that in particle model.

\section{References}

Kondo, E., Tanaka, H. and Kawagoshi, N., Detection of self-excited chatter caused by mode coupling(1st report, Study on criterion of detection), Transactions of JSME, Series C, Vol.64, No.625 (1998), pp413-418 (in Japanese).

Madoliat,R. , Hayati,S. and Ghalebahman,A.G. , Modeling and analysis of frictional damper effect on chatter suppression in a slender endmill tool , Jounal of Advanced Mechanical Design , Systems, and Manufacturing , Vol.5, No.2 (2011), pp.115-128.

Onozuka, H. , Utsumi, K. , Kato, T. , Takahashi, H. and Obikawa, T. , Optimal design of a damped tool arbor for heavy duty machining of giant parts, Journal of Advanced Mechanical Design, Systems, and Manufacturing, Vol.7, No.2 (2013), pp171-186.

Ota, H., Kito,M. and Handa.T., A study on primary chatter caused by mode coupling of machine tool structure, Transactions of JSME, Series C, Vol.73, No.726 (2007), pp208-214 (in Japanese).

Pratt,J.R. and Nayfeh,A.H. , Chatter control and stability analysis of a cantilever boring bar under regenerative cutting conditions , Philosophical Transactions of the Royal Society of London , Part A 359 (2001), pp759-792.

Rashid,A. and Nicolescu,C.M. , Design and implementation of tuned viscoelastic dampers for vibration control in milling, International Journal of Machine Tools and Manufacture, Vol.45, Issue.9 (2008), pp.1036-1053.

Rivin,E.I. , Kang,H. , Enhancement of dynamic stability of cantilever tooling structures , International Journal of Machine Tools \& Manufacture, Vol.32, No.4(1992), pp539-561.

Saffury,J. and Altus,E. , Optimized chatter resistance of viscoelastic turning bars , Journal of Sound and Vibration , Vol.324 (2009), pp26-39.

Semercigil, S.E. and Chen, L.A. , Preliminary computations for chatter control in endmilling , Journal of Sound and vibration, Vol.249, No.3 (2002), pp622-633

Seto,K. , Dynamic vibration absorber and its applications , CORONA PUBLISHING CO.,LTD. (2010) (in Japanese).

Seto,K. and Yamada,K. , An investigation on boring bars equipped with a dynamic absorber , Proc. of the 4th Int.C.P.E (1980), pp422-427.

Sims,N.D., Vibration absorbers for chatter suppression: A new analytical tuning methodology, Journal of Sound and Vibration, Vol.301 (2007), pp592-607.

Suzuki, N., Ikada, T. Hino, R. and Shamoto, E., Comprehensive study on milling conditions to avoid forced / self-excited chatter vibrations, Journal of the Japan Society for Precision Engineering, Vol.75, No.7 (2009), pp908-914 (in Japanese).

Tlusty, J. and Polacek,M., The stability of machine tool against self-excited vibrations in machining, ASME Proceeding of Engineering Research Conference, Pittsburgh (1963), pp454-465.

Tlusty, J., Machine dynamics, edited by R. I. King, Handbook of high speed machining technology, Chapman and Hall, New York (1985), pp48-153.

Tobias, S.A., Machine Tool Vibration (1965), Blackie.

Zhang, X. J., Xiong, C.H., Ding, Y., Feng, M.J. and Xiong, Y.L., Milling stability analysis with simultaneously considering the structural mode coupling effect and regenerative effect, International Journal of Machine Tools \& Manufacture, Vol.53 (2012), pp127-140. 